\title{
The effects of endoplasmic reticulum stress inhibition on vascular and cardiac EGFR signaling in hypertension
}

\author{
Nur B. Bal ${ }^{1}$, Sevtap Han ${ }^{1}$, Merve M. Tuglu², Orhan M. Uludag ${ }^{1}$, Emine Demirel-Yilmaz ${ }^{2}$

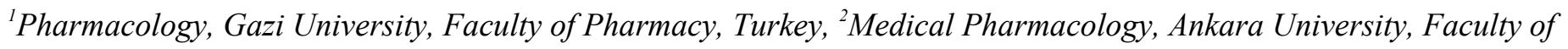 \\ Medicine, Turkey
}

Background: Vascular and cardiac complications are major causes of morbidity and mortality in hypertension. Epidermal growth factor receptor (EGFR) signaling associated with the development of vascular and cardiac dysfunction through activation of the extracellular signal-regulated kinase (ERK) and protein kinase B (Akt) signaling pathways. It is implicated that endoplasmic reticulum stress (ERS) plays a role in pathogenesis of hypertension. However, the relationship between EGFR signaling and ERS in hypertension has not determined yet. In the present study, we examined effects of ERS inhibition on the EGFR signaling in vascular and cardiac tissues from hypertensive rats.

Methods: Hypertension was induced by deoxycorticosterone acetate (DOCA) injection (20mg/kg, twice a week) and $1 \%$ $\mathrm{NaCl}+0.2 \% \mathrm{KCI}$ by adding to drinking water following the unilateral nephrectomy in male 8-week-old Wistar albino rats. Blood pressure was measured using tail-cuff method for 12 weeks. ERS inhibitor tauroursodeoxycolicacid (TUDCA) $(150 \mathrm{mg} / \mathrm{kg} /$ day $)$ was given by intraperitoneal injection last four week. At the end of treatment, abdominal aorta (AA), carotid artery (CA) and left ventricle (LV) tissues were removed and vascular tissues were incubated with phenylephrine. Then, expressions of EGFR, ERK, p-ERK, Akt, p-Akt were examined by Western Blotting.

Results: TUDCA treatment significantly reduced systolic blood pressures in hypertensive rats. EGFR expressions in AA and LV were similar in all groups. While EGFR expression in CA was increased in hypertensive group, TUDCA treatment reduced it. In AA, ERK expression was decreased by hypertension but, $\mathrm{p}$-ERK expression was not changed. In CA and LV, ERK and p-ERK expressions were similar in all groups. Akt and p-Akt expression were attenuated in AA isolated from hypertensive rats. p-Akt expression was augmented with phenylephrine treatment but, Akt expression in CA was not altered by hypertension. Akt and p-Akt expressions in LV were not changed by hypertension. TUDCA treatment did not affect Akt, p-Akt, ERK and p-ERK expressions in all tissues.

Conclusions: In hypertension, expressions of molecules that play a role in mitogenic pathway were changed in vascular wall but not cardiac tissue. Our results demonstrate that ERS inhibition can affect EGFR signaling and it may be a therapeutic strategy to prevent vascular complications in hypertension. 